\title{
Clinical Features, Laboratory Findings and Complications of Scrub Typhus in South Indian Children
}

\author{
Manjunath VG' ${ }^{\text {, Hedda }} \mathrm{P}^{2}$, Vijaykumar GS $^{3}$, Kumar JK ${ }^{4}$, Murthy $\mathrm{S}^{5}$
}

\begin{abstract}
Introduction: Scrub typhus clinical features are non-specific and resemble other tropical infections like malaria, dengue and typhoid fever. Therefore appropriate gold standard laboratory tests are necessary to confirm the diagnosis of scrub typhus. Aim of this study was to determine the incidence, clinical features, laboratory data and complications of scrub typhus in South Indian Children. Materials and Methods: Children with fever of more than seven days who were tested negative for common tropical infections were subjected to IgM-IFA for scrub typhus. Results: Out of 857 children, 74 were eligible for IFA test. Out of these, 27(3.1\%) tested positive for scrub typhus. Clinical features included hepatomegaly (96.3\%), generalized lymphadenopathy $(81.5 \%)$, splenomegaly (81.5\%), hypotension $(59.3 \%)$, rash $(14.8 \%)$, eschar $(7.4 \%)$, thrombocytopenia $(66.7 \%)$, elevation of SGOT $(85.2 \%)$ and SGPT $(81.5 \%)$. Complications include hepatitis $(14.8 \%)$, pneumonia (14.8\%), myocarditis (14.8\%) meningoencephalitis (3.7\%) and MODS (3.7\%).Conclusion: Scrub typhus should be considered in the differential diagnosis of a febrile child having hepatosplenomegaly, lymphadenopathy, liver dysfunction and thrombocytopenia.
\end{abstract}

Key words: Scrub typhus, Immune fluorescence antibody test, Children

\section{Introduction}

$A_{\text {th }}^{\text {nnu }}$ nnually an estimated one million Scrub typhus cases occur throughout the world ${ }^{1,2}$. In tropical countries, Rickettsial diseases may mimic other acute febrile illnesses such as malaria, dengue and typhoid fever, which are difficult to differentiate on clinical examination alone $^{3}$. This poses a challenge as the management strategies are different and misdiagnosis can be life threatening. The commonly performed Weil-Felix test for scrub typhus is notoriously unreliable ${ }^{1}$. Therefore Immune fluorescence antibody (IFA) test is considered as the gold standard diagnostic test ${ }^{1}$. This study was undertaken to find the incidence of scrub typhus using IFA in children with acute febrile illness.
${ }^{1}$ Dr. Manjunath Vaddambal Gopalakrishna, MBBS. DCh., DNB. Associate Professor of Paediatrics, ${ }^{2} \mathrm{Dr}$. Hedda Suryaprakash, MBBS. MD Resident in Paediatrics, ${ }^{3} \mathrm{Dr}$. G Shankarappa Vijay Kumar, Professor of Microbiology, ${ }^{4} \mathrm{Dr}$. Kalenahalli Jagadish Kumar MBBS., MD. Professor of Paediatrics, ${ }^{5}$ Dr.Doreswamy Srinivasa Murthy, MBBS. MD. Professor of Paediatrics. All from the Jagadguru Sri Shivarathreeshwara Medical College, Jagadguru Sri Shivarathreeshwara University, Mysore, India.

\section{Address for correspondence \\ Dr. Manjunath VG \\ 35 Ist Main Jayalaxmipuram, \\ Mysore, India-570012 \\ Tel No; +91-9448047548 \\ E-mail: vghunsur@rediffmail.com}

Acknowledgements: We are thankful to Dr. Basavanagowdappa H, Dr. Narayanappa $D$ and Dr. Ravi M D for their support and encouragement for the study. We also thank Dr. Lokesh $\mathrm{K} S$ for helping in preparation of this article.

Funding: Nil

Conflict of Interest: None

Permission from IRB/Ethical Board: The study was approved by Institutional Ethical Committee of JSS Medical College

\section{How to cite}

Manjunath VG, Hedda P, Vijaykumar GS, Kumar JK, Murthy S. Clinical Features, Laboratory Findings and Complications of Scrub Typhus in South Indian Children. J Nepal Paediatr Soc 2017;37(1):21-24.

doi: http://dx.doi.org/10.3126/jnps.v37i1.16202

This work is licensed under a Creative Commons Attribution 3.0 License. 


\section{Material and Methods}

This was a prospective observational study conducted between December-2011 and November-2012. A total of 857 children in the age-group of 1-15 years with fever of seven days or more were admitted to JSS Hospital, Mysore. All were subjected to relevant investigations such as complete blood count, ESR, blood \& urine culture, Buffy coat smear for malaria, dengue serology, Widal test, Mantoux test and Chest X-ray.

Seventy-four children in whom these investigations did not yield any etiological diagnosis were subjected to IFA testing. Their demographic, clinical and laboratory parameters were recorded in a predesigned proforma. Blood samples $(5 \mathrm{ml})$ were tested for specific IgM antibodies using a commercial IFA kit (Fullers laboratories. USA) which utilized four strains (Gilliam, Karp, Kato, Boryong). IFA was considered positive if the $\lg \mathrm{M}$ titres were 1:64 or greater. Renal and liver function tests, abdominal and chest sonography was done in these children and treated with doxycycline. Depending upon the clinical need, other appropriate investigations like $\mathrm{ECHO}, \mathrm{CPK}-\mathrm{MB}, \mathrm{CSF}$ and blood gas analyses were performed.

\section{\# Aetiological diagnosis in 783 cases}

Upper respiratory tract infections in 154, Malaria in 18, Dengue in 267, Pneumonia/empyema/lung abscess in 89, Enteric fever (typhoid and paratyphoid fever) in 113, Tuberculosis in 21, Urinary Tract Infection in 42, Chikungunya fever in 15,Acute viral hepatitis (A and E) in 13, Infectious mononucleosis in 2, Systemic Lupus Erythematosis in 2, Juvenile Idiopathic Arthritis in 4, Leukaemia in 2, Discharged Against Medical Advice 22 and others 19 .

\section{Results}

Seventy four of $857(8.6 \%)$ children remained undiagnosed after evaluation for Malaria, Enteric fever, Dengue, Tuberculosis, Acute respiratory and Urinary infections[Fig 1]. Out of these 74 children, 27 (36.4\%) tested positive for O.tsutsugamushi by IFA[Fig 2] and all belonged to Gilliam strain. Overall $3.15 \%$ of acute febrile children were positive for O.Tsutsugamushi in the present study. Among the cases, 59.3\% were from rural area and $63 \%$ presented in winter season.

Headache was present in $48.1 \%$ while hypotension was recorded in $59.3 \%$ of cases. Hepatomegaly was found in $96.3 \%$, generalized lymphadenopathy and splenomegaly in $81.5 \%$ of cases each (Table 1). Thrombocytopenia was observed in 66.7 and a raised SGOT \& SGPT was seen in 85.2 and $81.5 \%$ respectively (Table 2 ). A clinical diagnosis of myocarditis was entertained in 6 children and 4 of them had elevated CK-MB levels (Table 2). All cases recovered without any squeal.

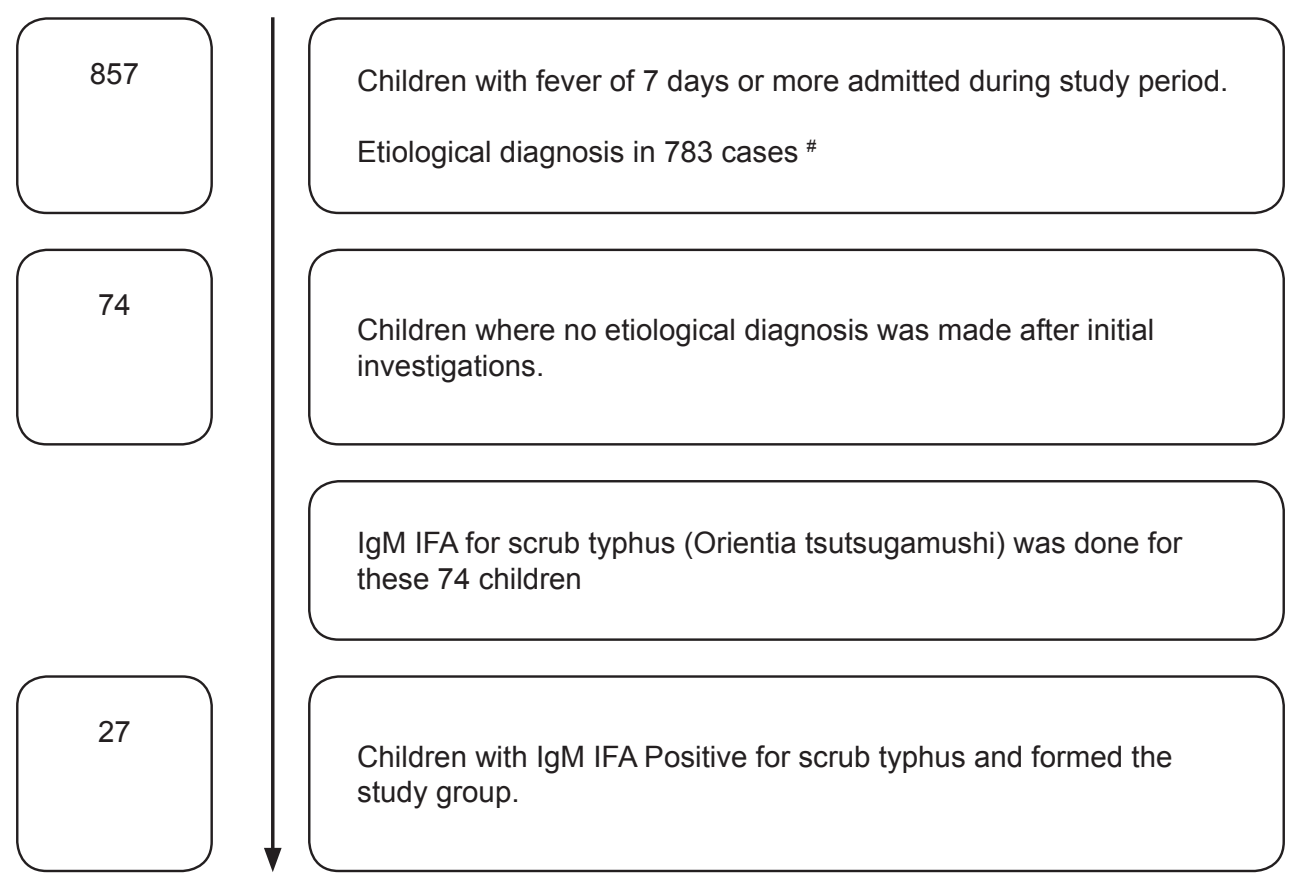

Fig 1: Flow Chart depicting recruitment of cases to the study 
Table 1: Clinical profile (symptoms and signs) of scrub typhus $(\mathrm{N}=27)$

\begin{tabular}{lc}
\hline Clinical features(Symptoms/History) & Number (\%) \\
\hline Fever & $27(100)$ \\
\hline Headache & $13(48.1)$ \\
\hline Abdominal pain & $10(37)$ \\
\hline Vomiting & $10(37)$ \\
\hline Myalgia & $9(33.3)$ \\
\hline Cough & $3(11.1)$ \\
\hline Joint pain & $2(7.4)$ \\
\hline Altered sensorium & $2(7.4)$ \\
\hline Signs & \\
\hline Hepatomegaly & $26(96.3)$ \\
\hline Lymphadenopathy & $22(81.5)$ \\
\hline Splenomegaly & $22(81.5)$ \\
\hline Hypotension & $16(59.3)$ \\
\hline Periorbital puffiness & $13(48.1)$ \\
\hline Conjunctival congestion & $9(33.3)$ \\
\hline Pedal oedema & $8(29.6)$ \\
\hline Ascites & $8(29.6)$ \\
\hline Rash & $4(14.8)$ \\
\hline Tachypnea & $3(11.1)$ \\
\hline Eschar & $2(7.4)$ \\
\hline
\end{tabular}

Table 2: Abnormal Laboratory parameters and complications in scrub typhus $(\mathrm{N}=27)$

\begin{tabular}{lc}
\hline \multicolumn{1}{c}{ Laboratory parameters } & Number (\%) \\
\hline Hematological & $18(66.7)$ \\
\hline Thrombocytopenia & $13(48.1)$ \\
\hline Anemia & $5(18.5)$ \\
\hline Leucocytosis & $1(3.7)$ \\
\hline Leukopenia & \\
\hline Biochemical & $23(85.2)$ \\
\hline AST(>40 IU/L) & $22(81.5)$ \\
\hline ALT(>40 IU/L) & $17(62.9)$ \\
\hline Serum sodium(<135) & $15(55.6)$ \\
\hline Serum Albumin(<3.5) & $5(18.5)$ \\
\hline Increased ALP & $2(7.4)$ \\
\hline Total Serum Bilirubin >1.5mg\% & \\
\hline Imaging & $9(33.3)$ \\
\hline Ascitis & $5(18.5)$ \\
\hline Pleural effusion & $4(14.8)$ \\
\hline Pneumonia & \\
\hline Complications\# & $4(14.8)$ \\
\hline Hepatitis [AST \& ALT>3 times & $4(14.8)$ \\
elevation] & \\
\hline Cardiac involvement & \\
\hline CPK-MB $>2$ times elevation= 2 (7.4) & \\
CPK-MB>4 times elevation=2 (7.4) & \\
\hline
\end{tabular}

\# Meningoencephalitis, Severe thrombocytopenia

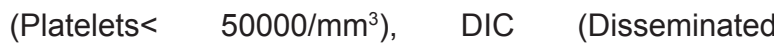
Intravascular Coagulation) \& MODS (Multi Organ Dysfunction Syndrome) were seen in one child each $(3.7 \%)$.

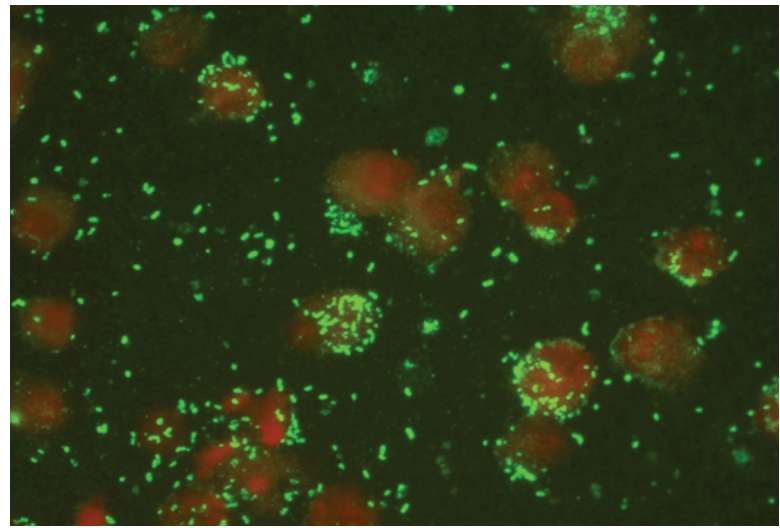

Fig 2: IFA positive reaction which appears as bright staining of short pleomorphic rod forms $[400 \mathrm{x}$ magnification]

\section{Discussion}

This study demonstrated the incidence of scrub typhus in children with more than one week of fever to be $3.15 \%$. More than half were from rural area and nearly two thirds were seen in winter months. Similar geographic and seasonal trend have been reported by other authors ${ }^{1,4,5}$. This could be due to more vegetation and conducive environment for the chigger-mites to thrive in rural areas.

The clinical picture in scrub typhus is quite nonspecific, necessitating clinicians to have high index suspicion for the diagnosis. Headache, macular rash, conjunctival hyperaemia noted in our study is common observation in scrub typhus $\mathrm{s}^{6,7,8}$. None of our cases had history of tick exposure. Characteristic feature of scrub typhus is eschar, which was seen in less than $10 \%$ of our cases. However other authors have reported eschar between $10-92 \%$ of their cases ${ }^{2}$. Though it is difficult to ascertain the cause for such a low incidence of eschar in our children, it could be due to the different strain of the organism, and possibility of various ethnic populations responding differently to mite bite ${ }^{2}$.

Features of capillary leak such as pedal oedema and peri-orbital puffiness were seen in many of our patients. Similar findings have been reported by Somashekar and Gurung et al ${ }^{6,7}$. Hypotension was noted in more than half of our patients confusing the diagnosis with Dengue fever. Only one child presented in shock which is in contrast to another study from Northern India which has reported shock in more than quarter of their patients ${ }^{9}$.

The incidence of hepatosplenomegaly, lymphadenopathy, thrombocytopenia and hypoalbuminemia noted in our study was similar to other studies $4,6,9,10,11,12$. Kim et al observed significant 
hypoalbuminemia in severe scrub typhus patients ${ }^{13}$. Scrub typhus closely resembles dengue infection resulting in diagnostic confusion. The presence of an eschar, tender lymphadenopathy, splenomegaly and persistence of fever after the recovery from shock helps to distinguish Rickettsial infection from dengue ${ }^{3,9}$.

In our study, $14.8 \%$ of children with scrub typhus developed pneumonia. $37 \%$ of scrub typhus children developed Pneumonitis in a study by Sirisanthana et $\mathrm{al}^{8}$. Hepatitis was observed in $14.81 \%$ of children but none had jaundice. In a study by Yang et al, six out of 47 scrub typhus cases presented similar to viral hepatitis ${ }^{14}$. Myocarditis is rare and some children may have unrecognised mild myocarditis ${ }^{15}$.We had four children with elevated CPK-MB suggesting myocardial injury but all had normal ECHO. Jim et al reported myocarditis in $5 \%$ of children ${ }^{14}$. Meningo-encephalitis is a dreaded but rare complication in scrub typhus. We had only one child with this complication and a similar incidence is noted by Sirisantana et al in their study ${ }^{8}$.

\section{References}

1. World Health Organization. Regional Office for South East Asia. Frequently Asked Questions. Scrub Typhus. Available at: www.searo.who.int/entity/emerging diseases/CDS_faq_Scrub_Typhus.pdf (28 January 2016, date last accessed)

2. Varghese G M, Trowbridge $P$, Janardhanan J, et al. Clinical Profile and Improving Mortality Trend of Scrub Typhus in South India. Int J Infect Dis 2014;23:39-43. doi: 10.1016/j.ijid.2014.02.009

3. Hamaguchi S, Cuong NC, Tra DT, et al. Clinical and Epidemiological Characteristics of Scrub Typhus and Murine Typhus among Hospitalized Patients with Acute Undifferentiated Fever in Northern Vietnam. Am J Trop Med Hyg 2015;92:972-78. doi: 10.4269/ ajtmh.14-0806.

4. Huang CT, Chi H, Lee HC, Chiu NC, Huang FY. Scrub typhus in children in a teaching hospital in Eastern Taiwan,2000-2005.Southeast Asian J Trop Med Public Health.2009; 40:789-794.PMID:19842416

5. Narvencar K P S, Rodrigues S, Nevrekar RP, et al. Scrub typhus in patients reporting with acute febrile illness at a tertiary health care institution in Goa. Indian J Med Res 2012;136:1020-24. PMCID: PMC3612306

6. Somashekar HR, Moses PD, Pavithran S, et al. Magnitude and Features of Scrub Typhus and Spotted Fever in Children in India. J Trop Pediatr. 2006;52:228229.doi: 10.1093/tropej/fmi096

7. Gurung S, Pradhan J, Bhutia P Y. Outbreak of scrub typhus in the North East Himalayan regionSikkim: An emerging threat. Indian J Med Microbiol 2013;31:7doi:10.4103/0255-0857.108729
Indirect florescent antibody test for scrub typhus has an advantage of being useful in the first week of illness over traditional Weil-Felix test which is useful only in the second week of illness. Currently IFA is considered as gold standard serological test for scrub typhus ${ }^{1}$.

Limitation of our study is possibility of missing mild cases of scrub typhus that had fever of less than seven days duration. We did not demonstrate raise in titres using paired sera of our subjects.

\section{Conclusion}

The incidence of scrub typhus using IFA was $3.15 \%$ in children with acute febrile illness in our study. The clinical and laboratory features can be confusing with Dengue and other tropical illness. Scrub typhus should be strongly considered in any child with non-relenting febrile illness with hepatosplenomegaly, lymphadenopathy, liver dysfunction and thrombocytopenia.

8. Sirisanthana V,Puthanakit T,Sirisanthana T. Epidemiologic, clinical and laboratory features of scrub typhus in thirty Thai children. Pediatr Infect Dis J 2003;22:341-45.doi:10.1097/01. inf.0000059400.23448.57

9. Kumar Bhat N, Dhar M, Mittal G, et al. Scrub Typhus in Children at a Tertiary Hospital in North India: Clinical Profile and Complications. Iran J Pediatr 2014;24:38792.PMCID:PMC4339561

10. Palanivel S, Nedunchelian K, Poovazhagi V, Raghunadan R, Ramachandran P. Clinical profile of scrub typhus in children. Indian J Pediatr 2012;79:1459462.doi: 10.1007/s12098-012-0721-0

11. .De Silva N, Wijesundara S, Liyanapathirana V, Thevanesam V, Stenos J. Scrub Typhus among Pediatric Patients in Dambadeniya:A Base Hospital in Sri Lanka. Am J Trop Med Hyg 2012;87:342-44.doi: 10.4269/ajtmh.2012.12-0170.

12. .Jim WT, Chiu NC, Chan WT, et al. Clinical manifestations, laboratory findings and complications of pediatric scrub typhus in eastern Taiwan. Pediatr Neonatol 2009;50:96-101. doi: 10.1016/S18759572(09)60043-7.

13. Kim DM, Kim SW, Choi SH, Yun NR. Clinical and laboratory findings associated with severe scrub typhus. BMC Infect Dis 2010;10:108.doi: 10.1186/14712334-10-108.

14. Yang $\mathrm{CH}$, Hsu GJ, Peng MY, Young TG. Hepatic dysfunction in scrub typhus. J Formos Med Assoc 1995;94:101-105.PMID:7613239

15. Sittiwangkul $R$, Pongprot $Y$, Silviliarat $S$, Oberdorfer $P$, Jittamala $P$, Sirisanthana V. Acute fulminant myocarditis in scrub typhus. Ann Trop Paediatr 2000;28:149-154. doi: $10.1179 / 146532808 \times 302189$. 